\title{
Millimeter-wave spectroscopy of hydantoin, a possible precursor of glycine ${ }^{\star}$
}

\author{
Hiroyuki Ozeki ${ }^{1}$, Rio Miyahara ${ }^{1}$, Hiroto Ihara ${ }^{1}$, Satoshi Todaka ${ }^{1}$, Kaori Kobayashi ${ }^{2,3}$, and Masatoshi Ohishi ${ }^{4}$ \\ ${ }^{1}$ Department of Environmental Science, Faculty of Science, Toho University, 2-2-1 Miyama, 274-8510 Funabashi, Japan \\ e-mail: ozeki@env.sci.toho-u.ac.jp \\ 2 Department of Physics, Faculty of Science, University of Toyama, 3190 Gofuku, 930-8555, Toyama, Japan \\ ${ }^{3}$ National Astronomical Observatory of Japan, 2-21-1 Oosawa, 181-8588 Mitaka, Japan \\ e-mail: kaori@sci.u-toyama.ac.jp \\ 4 Astronomy Data Center, National Astronomical Observatory of Japan, 2-21-1 Oosawa, 181-8588 Mitaka, Japan \\ e-mail: masatoshi.ohishi@nao.ac.jp
}

Received 11 October 2016 / Accepted 12 January 2017

\begin{abstract}
Context. Hydantoin (Imidazolidine-2, 4-dione, $\mathrm{C}_{3} \mathrm{H}_{4} \mathrm{~N}_{2} \mathrm{O}_{2}$ ) is a five-membered heterocyclic compound that is known to arise from prebiotic molecules such as glycolic acid and urea, and to give the simplest amino acid, glycine, by hydrolysis under acidic condition. The gas chromatography combined with the mass spectrometry of carbonaceous chondrites lead to the detection of this molecule as well as several kinds of amino acids.

Aims. The lack of spectroscopic information, especially on the rotational constants, has prevented us from conducting a search for hydantoin in interstellar space. If a rotational temperature of $100 \mathrm{~K}$ is assumed as the kinetic temperature of a star-forming region, the spectral intensity is expected to be at its maximum in the millimeter-wave region. Laboratory spectroscopy of hydantoin in the millimeter-wave region is the most important in providing accurate rest frequencies to be used for astronomical research.

Methods. Pure rotational spectra of hydantoin were observed in the millimeter-wave region using the frequency modulated microwave spectrometer at Toho University. Solid hydantoin was heated to around $150{ }^{\circ} \mathrm{C}$ to provide appropriate vapor pressure. Quantum chemical calculations suggest that the permanent dipole moment of this molecule lies almost along the $b$-molecular axis, so that spectral search for $b$-type R-branch transition has been conducted.

Results. Rotational and centrifugal distortion constants up to the fourth order for the ground vibrational state of hydantoin were accurately determined by measuring $161 b$-type transitions in the frequency range between 90 and $370 \mathrm{GHz}$. In addition, we succeeded in assigning 230 satellite lines, which were attributed to the two vibrationally excited states. The spectral intensity ratio of these lines indicates that these states correspond to the low-lying (approximately $150 \mathrm{~cm}^{-1}$ above the ground state) vibrational modes.

Conclusions. The frequency catalog of hydantoin in the millimeter-wave range was created for the ground state and for the two low-lying excited states, and are ideal for a future astronomical research. The $1 \sigma$ frequency accuracy is lower than $100 \mathrm{kHz}$ for the lines with upper-state energy below $200 \mathrm{~cm}^{-1}$, corresponding to a velocity resolution of $0.1 \mathrm{~km} \mathrm{~s}^{-1}$ at $300 \mathrm{GHz}$
\end{abstract}

Key words. line: identification - molecular data - ISM: molecules - submillimeter: ISM

\section{Introduction}

The search for prebiotic molecules in interstellar space has been attractive to many researchers for a long time. Among the building blocks of the constituent materials of life, the first report of a trial for the detection of glycine in the interstellar medium (ISM) with a radio telescope was published by Brown et al. (1979). For more than three decades since then, the detection of glycine, the simplest amino acid, has been unsuccessful (Hollis et al. 2003a, 2003b; Kuan et al. 2003; Snyder et al. 2005; Cunningham et al. 2007; Jones et al. 2007; Belloche et al. 2008, 2011); however, Garrod (2013) predicted that ALMA would be able to detect gas-phase glycine released from dust grains at kinetic temperatures above $100 \mathrm{~K}$. To avoid difficulties in detecting complex molecules toward hot cores, due to the high density of spectral lines, Jiménez-Serra et al. (2014) claimed that photo-desorbed

* The spectral line list of hydantoin is available at the CDS via anonymous ftp to cdsarc. u-strasbg. fr $(130.79 .128 .5)$ or via http://cdsarc.u-strasbg.fr/viz-bin/qcat?]/A+A/600/A44 glycine may instead be detectable in low-mass prestellar cores such as L1544.

Various kinds of formation mechanisms of glycine in the ISM have been proposed. A Strecker-type reaction (Strecker 1850) may be one of the potential mechanisms. In this reaction scheme, aminoacetonitrile $\left(\mathrm{NH}_{2} \mathrm{CH}_{2} \mathrm{CN}\right)$ and methanimine $\left(\mathrm{CH}_{2} \mathrm{NH}\right)$ are the key intermediate molecules, and both molecules have already been detected mainly by spectral observation in the millimeter-wave region (e.g., Belloche et al. 2008, 2011; Godfrey et al. 1973; Qin et al. 2010; Turner et al. 1999; Tenenbaum et al. 2010; Martin et al. 2006; Suzuki et al. 2016). We have extended spectroscopic information of these species to the terahertz region to make it possible to carry out future searches in higher frequency regions, since the spectral line intensities would reach a maximum in typical interstellar condition (Dore et al. 2012; Motoki et al. 2013, 2014). The column densities of these precursor molecules were reported to be on the order of $10^{14}-10^{16}$ molecules $\mathrm{cm}^{-2}$ (Belloche et al. 2008, 2011; Godfrey et al. 1973), whereas the upper limit of glycine has so far been estimated to be $1.4 \times 10^{17} \mathrm{~cm}^{-2}$ toward Sgr B2 LMH 
(Hollis et al. 2003b) and 8-121 $\times 10^{14} \mathrm{~cm}^{-2}$ toward OMC-1 (Hollis et al. 2003a). Rigorous evaluations of the consistency between the observational results and chemical models are needed, which may lead to a call for other formation mechanisms.

Another possible glycine precursor, hydantoin (imidazolidine-2, 4-dione, $\mathrm{C}_{3} \mathrm{H}_{4} \mathrm{~N}_{2} \mathrm{O}_{2}$ ), came to our attention since this molecule was detected in carbonaceous chondrites together with several kinds of amino acids by gas chromatography combined with mass spectrometry (Shimoyama \& Ogasawara 2002). Hydantoin, whose structure is shown in Fig. 1, is a five-membered heterocyclic compound; it is known to arise from prebiotic molecules such as glycolic acid and urea (e.g., M.-Salván \& M.-Yaseli 2012), and to give the simplest amino acid glycine by hydrolysis under acidic condition (e.g., Marcellus et al. 2011).

From the molecular structural point of view, hydantoin itself is not a chiral molecule since one of the carbon atoms constituting the five-membered ring (C5 position) is bonded with two hydrogen atoms. The asymmetric substitution of either of the hydrogen atoms to the corresponding carbon atom (the so-called 5-substituted hydantoins) introduces enantiomerism.

Spectroscopic information on hydantoin is fairly limited. The molecular structure of solid hydantoin was derived by X-ray crystallography (Yu et al. 2004). Infrared and Raman spectroscopy in the solid phase were conducted to observe vibrational spectra for normal (Lebedev et al. 1969; Saito \& Machida 1978; Christiani et al. 1985; Sohar 1968; Ildiz et al. 2012) and for N- and C-deuterated isotopologues (Saito \& Machida 1978). Low-lying vibrational modes were identified by Saito \& Machida (1978). Recently, infrared spectra were recorded for matrix isolated hydantoin by Ildiz et al. (2013), but these low-lying vibrational states were not detected, even though theoretical calculations suggested their existence. Other computational studies were reported by using molecular mechanics and density functional methods to calculate molecular geometry, dipole moment, vibrational frequencies, etc. (e.g., Ildiz et al. 2012; Belaidi et al. 2015). To the best of our knowledge, no gas phase data have been obtained by high-resolution spectroscopy. Lack of millimeter-wave transition information prevents us from searching for hydantoin in the ISM.

In this paper we report pure rotational spectra of hydantoin observed in the millimeter-wave region for the first time. Molecular constants for the ground vibrational state and for the two low-lying vibrational excited states are provided. Spectral line catalogues for these three states provide accurate rest frequencies up to at least $300 \mathrm{GHz}$, where the line intensity is at their maximum at $100 \mathrm{~K}$. The estimated frequency accuracy is less than $<0.1 \mathrm{MHz}$, corresponding to a velocity resolution of $<0.1 \mathrm{~km} \mathrm{~s}^{-1}$ at $300 \mathrm{GHz}$.

\section{Experiment}

Commercially available hydantoin (Sigma Aldrich, 98\% purity) was used without further purification. The sample powder of approximately 20 grams was set inside a glass absorption cell (100 cm in length and $6 \mathrm{~cm}$ in diameter), which was then heated to $150{ }^{\circ} \mathrm{C}$. Hydantoin (melting point $220^{\circ} \mathrm{C}$ ) does not decompose at this temperature. The recorded spectra in the present study were observed when we heated the compound above $100{ }^{\circ} \mathrm{C}$, and were reproducible after repeated heating. The pressure of the cell was kept at around $1 \mathrm{~Pa}$.

All spectral lines were measured with the $23 \mathrm{kHz}$-source frequency modulated spectrometer at Toho University, Japan. The details of our spectrometer have been described extensively in

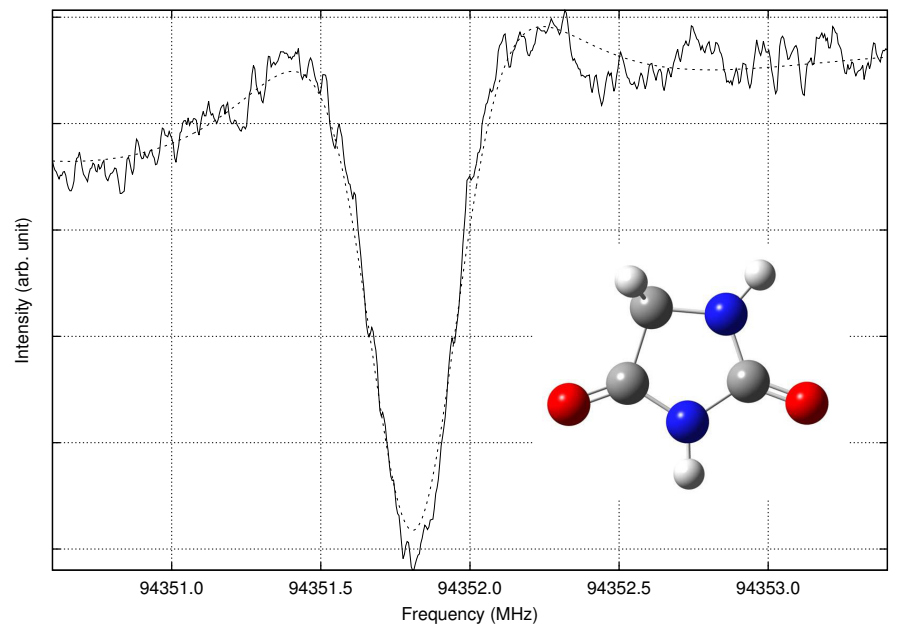

Fig. 1. Observed and fitted spectra of hydantoin (solid and dashed lines, respectively) at $93 \mathrm{GHz}\left(N_{K_{a} K_{c}}=27_{027} \leftarrow 26_{126}, N_{K_{a} K_{c}}=27_{127} \leftarrow\right.$ $26_{026}$,). The spectrum was recorded by integrating 400 scans at a $0.5 \mathrm{~Hz}$ repetition rate and a $1 \mathrm{~ms}$ time constant of the lock-in amplifier.

our previous study (Motoki et al. 2013). Millimeter-wave radiation in the frequency range of $90-370 \mathrm{GHz}$ was provided with the state-of-the-art frequency multiplier chains (VDI Inc.) driven by a microwave synthesizer (Agilent $8257 \mathrm{D}$ ). The InSb photo conductive detector that operates at $4 \mathrm{~K}$ (QFI-3BI, QMC) were used for detection. The $40 \mathrm{~dB}$ preamplified signal is detected at twice the modulation frequency, giving a second-derivative line shape. An example of the measured spectrum is shown in Fig. 1; it is clear that the signal-to-noise ratio of the spectra of hydantoin is sufficient. The frequency measurement error in this frequency region is approximately $30 \mathrm{kHz}$.

The molecular structure of hydantoin has been calculated by several computational studies (e.g., Belaidi et al. 2015) which are in close agreement with the structure obtained by X-ray crystallography (Yu et al. 2004). We also conducted the DFT calculations of hydantoin with the B3LYP/aug-cc-pVQZ basis set (Gaussian 09 et al. 2016) to obtain the dipole moment component along each molecular axis. Rotational A, B, and C constants of the molecule were obtained of 6567,2291 , and $1717 \mathrm{GHz}$, respectively. The permanent dipole moment lies almost along the molecular $b$-axis $(\mu(b)=2.655 \mathrm{D}$ and $\mu(a)=0.169 \mathrm{D})$. Hence a series of $b$-type R-branch transitions were expected to be predominantly observed in the millimeter-wave region.

\section{Spectral analysis}

The search for spectral lines in the frequency range between 138 and $149 \mathrm{GHz}$ showed that a bunch of intense lines appeared repeatedly with a frequency spacing of approximately $3340 \mathrm{MHz}$. The number of the observed intense lines in an individual bunch is larger than expected when we attribute them to the spectral lines in the ground vibrational state. As mentioned in the Introduction, data obtained using infrared and Raman spectroscopy (Saito \& Machida 1978), and via several theoretical calculations (Ildiz et al. 2012, 2013; Belaidi et al. 2015), suggest that there are at least two low-lying vibrational excited states (below 300 $\mathrm{K}$, or $200 \mathrm{~cm}^{-1}$ ) for hydantoin. Initial assignments were made possible by assuming that the observed intense lines are due to the $b$-type R-branch transition in the ground state and in two excited vibrtaional states. The observed data were analyzed by using the SPFIT/SPCAT suite of programs (Pickett 1991). The 
H. Ozeki et al.: Millimeter-wave spectroscopy of hydantoin

Table 1. Selected observed transition frequencies of hydantoin in its ground and excited vibrational states around $130 \mathrm{GHz}$.

\begin{tabular}{|c|c|c|c|c|c|c|}
\hline \multirow{2}{*}{$N_{K_{a^{\prime}} K_{c^{\prime}}}^{\prime} \leftarrow N_{K_{a} K_{c}}$} & \multicolumn{2}{|c|}{ Ground state } & \multicolumn{2}{|c|}{ Excited state 1} & \multicolumn{2}{|c|}{ Excited state 2} \\
\hline & Frequency & obs. - calc. & Frequency & obs. - calc. & Frequency & obs. - calc. \\
\hline $\begin{array}{l}36_{036} \leftarrow 35_{135} \\
36_{136} \leftarrow 35_{035}\end{array}$ & $125242.840(30)^{a}$ & -0.012 & $125452.478(30)$ & 0.005 & $125416.183(30)$ & -0.004 \\
\hline $\begin{array}{l}35_{134} \leftarrow 34_{233} \\
35_{234} \leftarrow 34_{233}\end{array}$ & $125145.367(30)$ & 0.009 & $125338.912(30)$ & -0.006 & $125304.957(30)$ & 0.011 \\
\hline $34_{232} \leftarrow 33_{331}$ & $125066.061(30)$ & 0.030 & $125243.414(30)$ & 0.061 & $125211.761(30)$ & 0.028 \\
\hline $34_{332} \leftarrow 33_{231}$ & $125066.423(30)$ & 0.045 & $125243.640(30)$ & -0.042 & $125212.140(30)$ & 0.072 \\
\hline $33_{330} \leftarrow 32_{429}$ & $125027.012(30)$ & 0.030 & $125187.891(30)$ & -0.005 & $125158.646(30)$ & -0.001 \\
\hline $33_{430} \leftarrow 32_{329}$ & $125024.476(30)$ & 0.020 & $125202.643(30)$ & -0.004 & $125173.614(30)$ & -0.014 \\
\hline $\begin{array}{l}37_{037} \leftarrow 36_{136} \\
37_{137} \leftarrow 36_{036}\end{array}$ & $128675.048(30)$ & 0.010 & $128890.609(30)$ & -0.004 & $128853.294(30)$ & -0.003 \\
\hline $\begin{array}{l}36_{135} \leftarrow 35_{234} \\
36_{235} \leftarrow 35_{234}\end{array}$ & $128577.240(30)$ & 0.069 & $128776.727(30)$ & 0.037 & $128741.668(30)$ & -0.020 \\
\hline $\begin{array}{l}35_{233} \leftarrow 34_{332} \\
35_{333} \leftarrow 34_{232}\end{array}$ & $128496.290(30)$ & 0.016 & $128679.576(30)$ & 0.011 & $128646.973(30)$ & 0.060 \\
\hline $34_{331} \leftarrow 33_{430}$ & $128454.243(30)$ & -0.009 & $128621.017(30)$ & -0.052 & $128590.816(30)$ & 0.005 \\
\hline $34_{431} \leftarrow 33_{330}$ & $128463.123(30)$ & 0.018 & $128629.520(30)$ & 0.028 & $128599.358(30)$ & -0.015 \\
\hline $\begin{array}{l}38_{038} \leftarrow 37_{137} \\
38_{138} \leftarrow 37_{037}\end{array}$ & $132107.167(30)$ & -0.020 & $132328.734(30)$ & 0.020 & $132290.337(30)$ & -0.032 \\
\hline $\begin{array}{l}37_{136} \leftarrow 36_{235} \\
37_{236} \leftarrow 36_{235}\end{array}$ & $132009.008(30)$ & 0.027 & $132214.458(30)$ & 0.000 & $132178.451(30)$ & 0.026 \\
\hline $\begin{array}{l}36_{234} \leftarrow 35_{333} \\
36_{334} \leftarrow 35_{233}\end{array}$ & $131926.523(30)$ & 0.000 & $132115.805(30)$ & 0.016 & $132082.136(30)$ & 0.035 \\
\hline $35_{332} \leftarrow 34_{431}$ & $131881.030(30)$ & -0.031 & $132053.875(30)$ & 0.048 & $132022.529(30)$ & -0.017 \\
\hline $35_{432} \leftarrow 34_{331}$ & $131886.048(30)$ & -0.050 & $132058.577(30)$ & -0.033 & $132027.425(30)$ & 0.014 \\
\hline $\begin{array}{l}39_{039} \leftarrow 38_{138} \\
39_{139} \leftarrow 38_{038}\end{array}$ & $135539.317(30)$ & 0.019 & $135766.763(30)$ & -0.014 & $135727.337(30)$ & -0.039 \\
\hline $\begin{array}{l}38_{137} \leftarrow 37_{236} \\
38_{237} \leftarrow 37_{236}\end{array}$ & $135440.818(30)$ & 0.034 & $135652.199(30)$ & -0.018 & $135615.154(30)$ & 0.002 \\
\hline $\begin{array}{l}37_{235} \leftarrow 36_{334} \\
37_{335} \leftarrow 36_{234}\end{array}$ & $135356.938(30)$ & 0.018 & $135552.160(30)$ & 0.001 & $135517.445(30)$ & 0.009 \\
\hline $36_{333} \leftarrow 35_{432}$ & $135307.830(30)$ & -0.012 & $135486.609(30)$ & 0.028 & $135454.219(30)$ & -0.049 \\
\hline $36_{433} \leftarrow 35_{332}$ & $135310.636(30)$ & -0.055 & $135489.257(30)$ & -0.025 & $135456.952(30)$ & -0.065 \\
\hline
\end{tabular}

Notes. Hyperfine splitting due to the nitrogen nucleus was not resolved. Units are in MHz. ${ }^{(a)}$ Experimental frequency accuracy in the present study.

rotational constants and the fourth order centifugal distortion constants were successfully obtained by using the conventional Watson S-reduced Hamiltonian for an asymmetric top molecule. Some of the observed and calculated frequencies are given in Table 1 . The number of measured lines in the present study and the fitted lines, as well as the determined molecular constants for the ground state and the two excited vibrational states, are summarized in Table 2. The standard deviation of the fit was 39 $\mathrm{kHz}$, which is of the same order as our experimental frequency accuracy.

\section{Results and discussion}

We measured pure rotational spectra of hydantoin in its vibrational ground state and in two excited states up to $370 \mathrm{GHz}$. Fundamental frequencies of the two low-lying vibrational modes in the gas phase have not been directly observed yet. Ildiz et al. $(2012,2013)$ presented theoretically predicted infrared spectra of hydantoin by using the DFT(B3LYP) and MP2 levels of approximation, and showed that Ar-matrix isolated infrared spectra observed above $500 \mathrm{~cm}^{-1}$ were fully assigned with the help of the above information. Their calculation also showed that there are two low-lying vibrational modes below $200 \mathrm{~cm}^{-1}$, both of which are designated to be torsional motions of a five-membered ring. These low-lying modes were also reported by other computational studies (Ildiz et al. 2013; Belaidi et al. 2015). However, the vibrational frequencies of these modes strongly depend on the level of approximation and on the basis sets. For example, our calculation gave the vibrational energies of the two lowest modes to be $55.2 \mathrm{~cm}^{-1}$ and $146.7 \mathrm{~cm}^{-1}$, while the corresponding values of $29 \mathrm{~cm}^{-1}$ and $133 \mathrm{~cm}^{-1}$ were calculated by Ildiz et al. (2013), and $128 \mathrm{~cm}^{-1}$ and $144 \mathrm{~cm}^{-1}$ by Belaidi et al. (2015). We see a large discrepancy in frequency especially for the lowest mode, which is presumably due to a large anharmonicity of the potential surface.

We compared the spectral intensity of the same observed rotational transitions in the vibrationally excited states relative to that in the ground state, as shown in Fig. 2. Observed relative intensities were $1,0.60$, and 0.53 at $420 \mathrm{~K}$ for the ground state, vibrationally excited state 1 , and excited state 2 , respectively. 
Table 2. Molecular constants of hydantoin in its vibrational ground state and two excited states.

\begin{tabular}{lccc}
\hline \hline Parameter & Ground state & \multicolumn{1}{c}{ Excited state 1 } & Excited state 2 \\
\hline$A(\mathrm{MHz})$ & $6537.73998(70)$ & $6515.909(53)$ & $6520.377(44)$ \\
$B(\mathrm{MHz})$ & $2291.37582(57)$ & $2293.8411(72)$ & $2293.1771(56)$ \\
$C(\mathrm{MHz})$ & $1716.471265(67)$ & $1719.454978(126)$ & $1718.943561(247)$ \\
$\Delta_{J}(\mathrm{kHz})$ & $0.095338(167)$ & $0.08369(233)$ & $0.09135(290)$ \\
$\Delta_{J}(\mathrm{kHz})$ & $0.15923(69)$ & $0.408(72)$ & $-0.212(174)$ \\
$\Delta_{K}(\mathrm{kHz})$ & $2.25266(83)$ & $2.76(35)$ & $6.06(159)$ \\
$\delta_{J}(\mathrm{kHz})$ & $0.025701(83)$ & $0.01940(117)$ & $0.02305(145)$ \\
$\delta_{K}(\mathrm{kHz})$ & $0.28008(144)$ & $0.3031(254)$ & $0.056(106)$ \\
\hline \# of measured lines & 161 & 114 & 116 \\
\hline rms & \multicolumn{3}{c}{$39 \mathrm{kHz}$} \\
\hline
\end{tabular}

Notes. Values in parentheses denote one standard deviation and apply to the last digits of the constants.
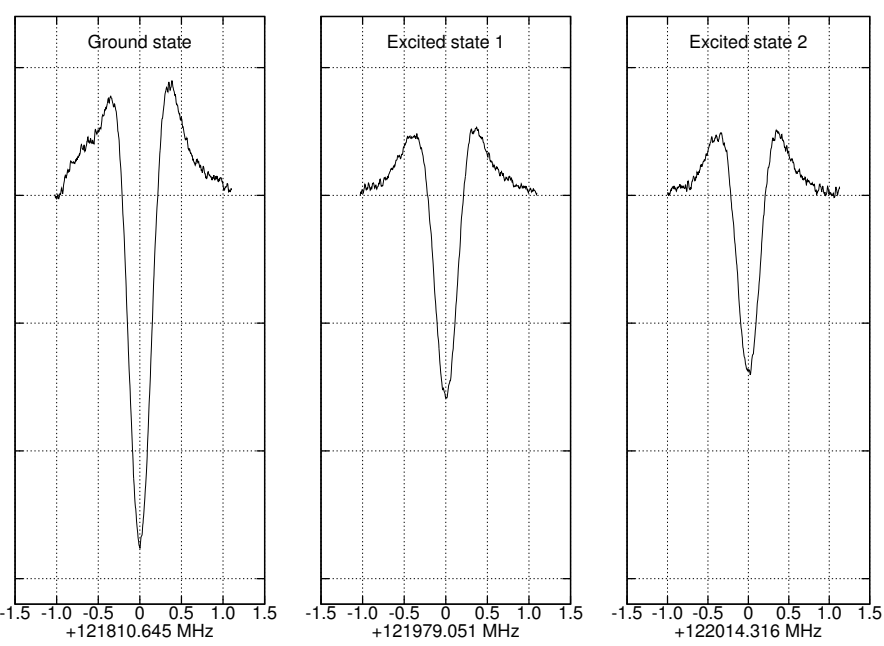

Fig. 2. Comparison of spectral intensity of hydantoin in its vibrational ground state (left) and two excited states (center and right) at $150^{\circ} \mathrm{C}$, observed at $121 \mathrm{GHz}\left(N_{K_{a} K_{c}}=35_{035} \leftarrow 34_{134}, N_{K_{a} K_{c}}=35_{135} \leftarrow\right.$ $\left.34_{0}{ }_{34}\right)$. Each spectrum was recorded by integrating 400 scans with a $0.5 \mathrm{~Hz}$ repetition rate and a $1 \mathrm{~ms}$ time constant of the lock-in amplifier.

The vibrational energy for these excited states are estimated to be 140 and $170 \mathrm{~cm}^{-1}$ for excited states 1 and 2, respectively. Any other series of spectral lines with larger intensities than those of these assigned excited states were not found in the present study; therefore, these two excited states must be due to the two lowest vibrational excited states.

The vibration-rotation partition functions of hydantoin for several temperatures were calculated by taking the two lowest vibrational excited states into account; they are listed in Table 3. With our calculated dipole moment $(\mu(b)=2.655 \mathrm{D}$ and $\mu(a)=0.169 \mathrm{D})$, and the molecular constants obtained in the present study, the spectral intensity distribution is estimated and is shown in Fig. 3. The main spectral pattern consists of a series of $b$-type transitions, whose intensities are reach their maximum at around $300 \mathrm{GHz}$, at a temperature of $100 \mathrm{~K}$, as shown in Fig. 3.

Given the partition function and the dipole moment along the $b$-axis for hydantoin, the expected spectral intensity can be calculated when we conduct a search for this molecule in the interstellar space. The integrated intensity for the low- $K_{a} b$-type

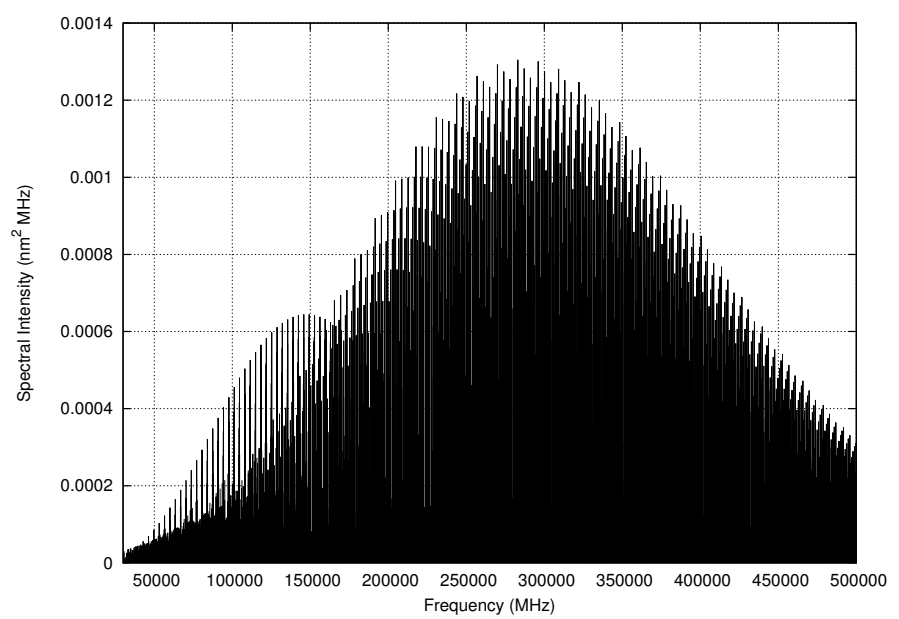

Fig. 3. Spectral intensity distribution of hydantoin at $100 \mathrm{~K}$.

Table 3. Vibration-rotation partition function of hydantoin.

\begin{tabular}{cr}
\hline \hline Temperature $(\mathrm{K})$ & \\
\hline 300 & 352393 \\
225 & 206091 \\
150 & 94144 \\
100 & 42631 \\
75 & 24781 \\
37.5 & 7725 \\
18.75 & 2703 \\
9.375 & 957 \\
\hline
\end{tabular}

Notes. Tabulated are vibration-rotation partition functions, for which the two lowest vibrational excited states are considered (see text).

R-branch transition $\left(N_{K_{a} K_{c}}=39_{039} \leftarrow 38_{138}, N_{K_{a} K_{c}}=39_{139} \leftarrow\right.$ $38_{0} 38$ at $135.5 \mathrm{GHz}$ ) is estimated to be $0.04-4 \mathrm{~K} \mathrm{~km} / \mathrm{s}$, assuming a column density of $10^{13}-10^{15} \mathrm{~cm}^{-2}$.

\section{Conclusions}

Pure rotational spectral lines of hydantoin in its ground vibrational state and the two lowest vibrational excited states were observed for the first time. The obtained millimeter data were 
analyzed and the molecular constants were obtained. The rotational partition function including the partition function of the two lowest vibrational excited states at various temperatures were calculated. The frequency error for spectral lines with upper-state energy below $200 \mathrm{~cm}^{-1}$ is $<100 \mathrm{kHz}$, corresponding to a velocity resolution of $0.1 \mathrm{~km} \mathrm{~s}^{-1}$ at $300 \mathrm{GHz}$, where the spectral line intensities are at their maximum with the temperature condition of $100 \mathrm{~K}$. Our provided spectral line list will be cataloged in a spectral line database (Toyama Microwave Atlas, ToyaMA $)^{1}$, also available at the CDS, and is valid for astronomical searches for hydantoin with millimeter to submillimeterwave telescopes such as ALMA.

Acknowledgements. H.O. thanks the Futaba Electronics Memorial Foundation for its financial support in constructing the spectrometer at Toho University. K.K. thanks Koichi Nozaki for helping ab initio calculations. This study was supported by JSPS KAKENHI (Grants-in-Aid for Scientific Research), grant numbers JP15H03646, JP15K05027.

\section{References}

Allegrini, M., Johns, J. W. C., \& McKellar, A. R. W. 1978, J. Chem. Phys., 70, 2829

Belaidi, S., Bouchlaleg, L., Harkati, D., \& Salah, T. 2015, Res. J. Pharm. Biol. Chem. Sci. 6, 861

Belloche, A., Menten, K. M., Comito, C., et al. 2008, A\&A, 482, 179

Belloche, A., Menten, K. M., Comito, C., et al. 2011, A\&A, 492, 769

Brown, R. D., Godfrey, P. D., Storey, J. W. V., et al. 1979, MNRAS, 186, 5

Buhl, D., \& Snyder, L. E. 1971, ApJ, 163, L47

Cheung, A. C., Rank, D. M., Towns, C. H., Thornton, D. D., \& Welch, W. J. 1968, Phys. Rev. Lett., 21, 1701

Cheung, A. C., Rank, D. M., Towns, C. H., Thornton, D. D., \& Welch, W. J. 1969, Nature, 221, 626

Christiani, F., Devillanova, F. A., Diaz, A., Isaia, F., \& Verani, G. 1985, Spectrochim. Acta A, 41, 487

Cummins, S. E., Linke, R. A., \& Thaddeus, P. 1986, ApJS, 60, 819

Cunningham, M. R., Jomes, P. A., Godfrey, P. D., et al. 2007, MNRAS, 376, 1201

Dore, L., Bizzochi, L., Degli Esposti, C., \& Gauss, J. 2010, J. Mol. Spectr., 263, 44

Dore, L., Bizzochi, L., \& Degli Esposti, C. 2012, A\&A, 544, A19

Duxbury, G. Kato, H., \& Le Lerre, M. L. 1981, Discuss. Faraday Soc., 71, 97

Gaussian 09, Frisch, M. J., Trucks, G. W., et al. 2016, Revision C.01, Inc., Wallingford CT

Garrod, \& R. T. 2013, ApJ, 765, 60
Godfrey, P. D., Brown, R. D., Robinson, B. J., \& Sinclair, W. M. 1973, ApJ, 13, L119

Gordy, W., \& Cook, R. L. 1984, Microwave Molecular Spectra, 3rd edn. (New York: Wiley)

Halonen, L., \& Duxubury, G. 1985, J. Chem. Phys., 83, 2078

Hamada, Y., Hashiguchi, K., Tsuboi, M. Koga, Y., \& Kondo, S. 1984, J. Mol. Spectr., 105, 70

Hollis, J. M., Lovas, F. J., \& Jewell, P. R. 2000, ApJ, 540, L107

Hollis, J. M., Pedelty, J. A., Snyder, L. E., et al. 2003a, ApJ, 588, 353

Hollis, J. M., Pedelty, J. A., Boboltz, D. A., et al. 2003b, ApJ, 596, L235

Ildiz, G. O., Boz, I., \& Unsalan, O. 2012, Opt. Spectr., 112, 665

Ildiz, G. O., Nunes, C. M., \& Fausto, R. 2013, J. Phys. Chem. A, 117, 726

Jiménez-Serra, I., Testi, L. Caselli, P., \& Viti, S. 2014, ApJ, 787, L33

Johnson, D. R., \& Lovas, F. J. 1972, Chem. Phys. Lett., 15, 65

Jones, P. A., Cunningham, M. R., Godfrey, P. D., \& Cragg, D. M. 2007, MNRAS, 374,579

Kirchhoff, W. H., Johnson, D. R., \& Lovas, F. J. 1973, J. Phys. Chem. Ref. Data, 2,1

Krause, H., Sutter, D. H., \& Palmer, M. H. 1989, Z. Naturforsch., 44a, 1063

Kuan, Y.-J., Charnley, S. B., Huang, H.-C., Tseng, W.-L., \& Kisiel, Z. 2003, ApJ, 593,848

Lebedev, R. S., Chumakova, R. P., \& Yakimenko, V. I. 1968, Sov. Phys. J., 12, 116

Marcellus, P., Bertrand, M., Nuevo, M., Westall, F., \& d'Hendecourt, L. S. 2011, Astrobiology, 11, 847

Martin, S., Mauersberger, R., Martin-Pintado, J., Henkel, C., \& Garcia-Burillo, S. 2006, ApJ Suppl., 164, 450

Menor-Salván, C., \& Marín-Yaseli, M. R. 2012, Chem. Soc. Rev., 41, 5404

Motoki, Y., Tsunoda, Y., Ozeki, H., \& Kobayashi, K. 2013, ApJS, 209, 23

Motoki, Y., Ozeki, H., \& Kobayashi, K. 2014, A\&A, 556, A28

Müller, H. S. P., Thornwirth, S., Roth, D. A., \& Winnewisser, G. 2001, A\&A, 370, L49

Müller, H. S. P., Schlöder, F., Stutzki, J., \& Winnewisser, G. 2005, J. Mol. Struct., 742,215

Pearson, Jr., R., \& Lovas, F. J. 1977, J. Chem. Phys., 66, 4149

Pickett, H. M. 1991, J. Mol. Spectr., 148, 371

Qin, S.-L., Wu, Y., Huang, M., et al. 2010, ApJ, 711, 399

Saito, Y., \& Machida, K. 1978, Bull. Chem. Soc. Jap., 51, 108

Shimoyama, A., \& Ogasawara, R. 2002, Orig. Life Evol. Biosph., 32165

Snyder, L. E., Buhl, D., Zuckerman, B., \& Palmer, P. 1969, Phys. Rev. Lett., 22, 679

Snyder, L. E., Lovas, F. J., Hollis, J. M., et al. 2005, ApJ, 619, 914

Sohar, P. 1968, Acta Chim. Sci. Hung., 57, 425

Strecker, A. 1850, Ann. Chem. Pharm., 75, 27

Suzuki, T., Ohishi, M., Hirota, T., et al. 2016, ApJ, 825

Tenenbaum, E. D., Dodd, J. L., Milam, S. N., Woolf, N. J., \& Ziurys, L. M. 2010, ApJ, 720, L102

Turner, B. E., Terzieva, R., \& Herbst, E. 1999, ApJ, 518, 699

Yu, F.-L., Y., Schwalbe, C. H., \& Watkin, D. J. 2004, Acta Crystallogr. C 2004, C60, 714

1 http://www.sci.u-toyama.ac.jp/phys/4ken/atlas/ 